\title{
Protocol
}

\section{Promoting independence in dementia: protocol for a feasibility trial of the PRIDE intervention for living well with dementia}

\author{
Emese Csipke ${ }^{1}$, Lauren Yates ${ }^{2}$, Esme Moniz Cook $^{3}$, Phuong Leung, \\ Georgina Charlesworth ${ }^{4}$, Holly Walton ${ }^{4}$, Linda Birt ${ }^{5}$, Martin Orrell ${ }^{2}$
}

\author{
${ }^{1}$ Division of Psychiatry, ${ }^{4}$ Department of Clinical, Educational and Health Psychology, University College London, UK \\ ${ }^{2}$ Institute of Mental Health, University of Nottingham, UK \\ ${ }^{3}$ Faculty of Health Sciences, University of Hull, Hull, UK \\ ${ }^{5}$ School of Health Sciences, University of East Anglia, Norwich, UK
}

Received: 13 June 2018

Accepted: 04 October 2018

\author{
*Correspondence: \\ Dr. Emese Csipke, \\ E-mail: e.csipke@ucl.ac.uk
}

Copyright: () the author(s), publisher and licensee Medip Academy. This is an open-access article distributed under the terms of the Creative Commons Attribution Non-Commercial License, which permits unrestricted non-commercial use, distribution, and reproduction in any medium, provided the original work is properly cited.

\begin{abstract}
Background: Dementia can lead to social exclusion, loss of identity and independence, due to deterioration in cognition and activities of daily living. The aim of the study is to investigate the feasibility of the Promoting Independence in Dementia (PRIDE) intervention, designed to facilitate independence in people with mild dementia. Methods: This is a mixed-methods feasibility trial of the PRIDE, in preparation for a future randomised controlled trial. Up to 50 people with dementia will be recruited. Demetia advisors will deliver the three session intervention. Quantitative outcomes will be taken at baseline and up to three months post baseline. Fidelity checklists will assess fidelity to the intervention. Qualitative implementation data will be gathered in a series of post-intervention semistructured interviews with staff and participants. This will include data to examine participant experiences of and engagement with the intervention, and other aspects of delivery such as recruitment of DAWs, fidelity and experiences of receiving and delivering the intervention. This study aims to establish and field test the PRIDE intervention; determine the recruitment rate of sites, providers and participants; assess fidelity in delivery of the intervention and engagement with people with dementia; assess the feasibility and acceptability of outcome measure data and assess the acceptability of the intervention by stakeholders.

Conclusions: There has been increased need for non-pharmacological interventions for mild dementia. The results of this feasibility study will allow us to plan for a definitive RCT of a three session dementia advisor led intervention for mild dementia.
\end{abstract}

Keywords: Dementia, Independence, Self-management, Decision-making, Agency, Dementia advice worker, Postdiagnostic support

\section{INTRODUCTION}

In the UK over 800,000 older people have dementia, which can lead to social exclusion, loss of identity and independence due to deterioration in cognition, activities of daily living, the double stigma of age and dementia, and the reduced capacity for social participation. ${ }^{1}$ The EU Joint Programme - Neurodegenerative Disease Research
(JPND) has highlighted the need for psychosocial interventions promoting social inclusion, dignity, and the positive contributions to society that people with dementia can make. ${ }^{2}$

People with early stage dementia are generally satisfied with their lives and feel their quality of life is 'good' but can, following a dementia-diagnosis experience stigma, 
feelings of hopelessness, insecurity, low self-esteem, and low confidence. ${ }^{3,4}$ Key concerns of people with dementia include loss of power in relationships with their family, the need to maintain a role outside the family, and a lack of basic information about diagnosis, prognosis, and care. ${ }^{5}$ Hobbies and interests are often lost early in the disease process, though there is evidence to suggest engaging in social, cognitive, and physical activities can slow down or prevent further cognitive deterioration and help preserve independence. ${ }^{6-9}$ Typically the person can become undermined by their diagnosis and the expectations and beliefs of others which may then lead to disengagement from life. ${ }^{10}$ Consequently, the person's 'sense of agency and self' in many spheres of everyday life, can also be undermined.

Supporters often misconstrue the values and preferences of people with dementia in relation to autonomy and consistently underestimate their decision-making ability. ${ }^{11}$ Responsibility for making decisions may be completely taken over by supporters, but many people with dementia see shared decision-making with family members as preferable. ${ }^{12}$ Those who lack opportunities to participate in decision making report feeling 'unheard'. 13 The assumption that the person lacks agency may also undermine their opportunities to initiate social action, and thus influence their own personal circumstances. ${ }^{14}$ These experiences may result in 'excess disability' whereby the person's reduced engagement with life is greater than would be expected for their impairment. ${ }^{15}$ The biopsychosocial model of dementia provides a theoretical framework to demonstrate how the person's social environment can reduce the risk and/or extent of excess disability experienced and optimise sense of self and independence. ${ }^{16}$

Due to the false assumption that there is little to offer people with dementia, and the stereotype that they "forget the information given to them" people with dementia may not be encouraged to take an active role in managing their care. ${ }^{17}$ Post-diagnostic support is critical to enhancing and maintaining the functional capacity of people with dementia, and helping them continue to engage in life, with lack of effective or insufficient support being linked to failure to prioritise diagnosis rate. ${ }^{18}$ The utility of dementia advisory services was shown in a recent independent assessment of improvements in dementia care and support, which reported that the most improved clinical commissioning groups (CCG) were those which offered dementia advisory services. ${ }^{18}$

\section{Aims}

The primary aim of the proposed study is to investigate the feasibility of the promoting independence in dementia (PRIDE) intervention delivered by DAWs designed to help facilitate the independence of people who have recently been diagnosed with dementia.
The study aims to determine:

- The acceptability of and engagement with the intervention

- Barriers and facilitators of the intervention

- The acceptability of outcome measures

- Recruitment rate and retention

- Fidelity of delivery of the intervention.

\section{METHODS}

The study is a mixed-methods feasibility trial of the PRIDE intervention. It will be non-randomised with all participants receiving the intervention. The study will integrate process evaluation, by assessing fidelity from the point of view of DAWs, persons with dementia and researchers. Engagement will be assessed from point of view of participants (person with dementia). The quantitative components of the trial will comprise of outcome assessments at baseline and immediately post intervention, intervention fidelity checklists, measurement of support required by the dyad (e.g., number of telephone support contacts, extra sessions required), and measurement of support required by the DAWs to deliver the intervention (e.g., number of support contacts and feedback about their training). Qualitative data on implementation, feasibility, and overall design of the intervention will be gathered from participants, supporters and DAWs in a series of semistructured interviews and focus groups, which will take place when the intervention is complete.

\section{Inclusion and exclusion criteria}

Up to 50 people with dementia and supporters (family, or friend) will be recruited across up to five sites in the UK.

\section{People with dementia}

People with dementia will meet the following eligibility criteria; community dwelling adults with a diagnosis of mild dementia. ${ }^{19}$ In order to be eligible, people with dementia will score $0.5-1$ on the clinical dementia rating scale (CDR), indicating mild dementia and who have a supporter (see below) willing to participate in the study alongside them. ${ }^{20}$ Participants must be able to read and communicate verbally in English, and able to provide informed consent to participate in accordance with the Mental Capacity Act Department of Health. ${ }^{21}$

\section{Supporters}

The protocol and intervention are using the term supporter rather than carer. Participants will be managing well independently and the term denotes support as being part of an ordinary relationship with reciprocity. Supporters must be unpaid and in regular contact with the person for a minimum of three hours per week. They will need to be able to communicate in English. 


\section{Dementia advice workers}

Dementia advice workers (DAWs) delivering the intervention will be health, social care, or voluntary sector staff working in or alongside memory clinics. Managers of participating organisations will be asked to refer suitable members of staff who are able to read and communicate verbally in English, able to attend training sessions, and are available to deliver the intervention to one or more participants.

\section{Sample size}

We aim to recruit up to 50 dyads and from up to five sites.

\section{Procedure}

Research staff will collaborate with memory services and National Health Service (NHS), local authority, and voluntary organisations such as Dementia UK, the Alzheimer's Society, Age UK, and Join Dementia Research (JDR; a national register where individuals, regardless of age or diagnosis can apply to express an interest in taking part in dementia research, potential participants are then matched to appropriate studies) to recruit participants to the study. Eligible participants from the JDR register will be sent the information sheet and sheet and researcher contact details as well as being informed/asked via JDR pathways if they are interested in taking part.

People with dementia and their supporters will be provided with study information when they express interest in the study. If they agree to participate, a member of the research team will visit them to seek written informed consent and then complete baseline measures with the person and their supporter. Participants will then be introduced to a local DAW who has been trained in the intervention protocol to deliver PRIDE programme. Participants and supporters will complete a follow up assessment up to three months after baseline assessment. Participants taking part in interviews or focus groups will sign a new consent form. Participants will be told that they can withdraw at any point without having to give a reason why, and without affecting the care they receive. A sample of persons with dementia, supports and DAWs will be invited to participate in interviews and focus groups after they have completed the intervention (Figure 1).

\section{Ethical approval}

Ethical approval was obtained through East Midlands Nottingham 1 Research Ethics Committee (ref no. 16/EM/0044). Participants will be fully informed of the potential benefits and risks associated with the study. People with dementia will be in the mild stages of dementia, and will have the capacity to be able to provide informed consent, provided appropriate care is taken to explain the study. All information sheets, consent forms and recruitment material have been approved, and will be unique to participation in the intervention, interviews or focus groups. Safety procedures for researchers in the UK will follow standard guidelines and potential risks will be minimal. Reporting procedures for serious adverse events (SAEs) are in place and will be reported to the Chief Investigator.

\section{Development of the intervention}

The development and feasibility assessment of the PRIDE intervention follows the Medical Research Council (MRC) guidance for complex interventions incorporating the additional refined methodology (e.g., analysis of practical issues impacting fidelity) in order to minimise risk of implementation error. ${ }^{22}$

The evidence used to inform the development of the first draft of the manual and intervention was derived from the PRIDE team's expertise and work including; a literature review of the effectiveness of psychosocial interventions to promote and maintain independence in early-stage dementia, a scoping review of social participation in people with dementia living in the community, evidence from the English Longitudinal Study of Ageing (ELSA) cohort about the links between loneliness and cognitive decline, the role of physical activity/exercise interventions, and the use of computers and the internet in cognitive function. ${ }^{23-27}$ The research team developed a first draft of the intervention and manual with input from people with dementia, supporters, DAWs, older people, and healthcare professionals, consulting these groups in a series of informal meetings, and via email. The manual was then updated based on consultation feedback to create a second draft, which will be tested in this feasibility study. In the final stage of the development of the intervention the feasibility trial data will be used in conjunction with further stakeholder consultation to make any final amendments to the intervention before a large scale RCT is launched to evaluate its effectiveness.

\section{PRIDE intervention}

The intervention aims to promote independence and facilitate the person's access to opportunities to live well with dementia by:

- Enabling the person to maintain a cognitively, physically, and socially active lifestyle

- Helping the person to optimise the resources they have (e.g., cognitive skills, social networks) by finding a balance in activities based on the selection, optimisation with compensation (SOC) framework ${ }^{28}$

- Aiding the person to have a healthy lifestyle (e.g., weekly exercise)

- $\quad$ Signposting to local services and resources

- Helping the person maintain their social roles and agency in everyday decision-making 


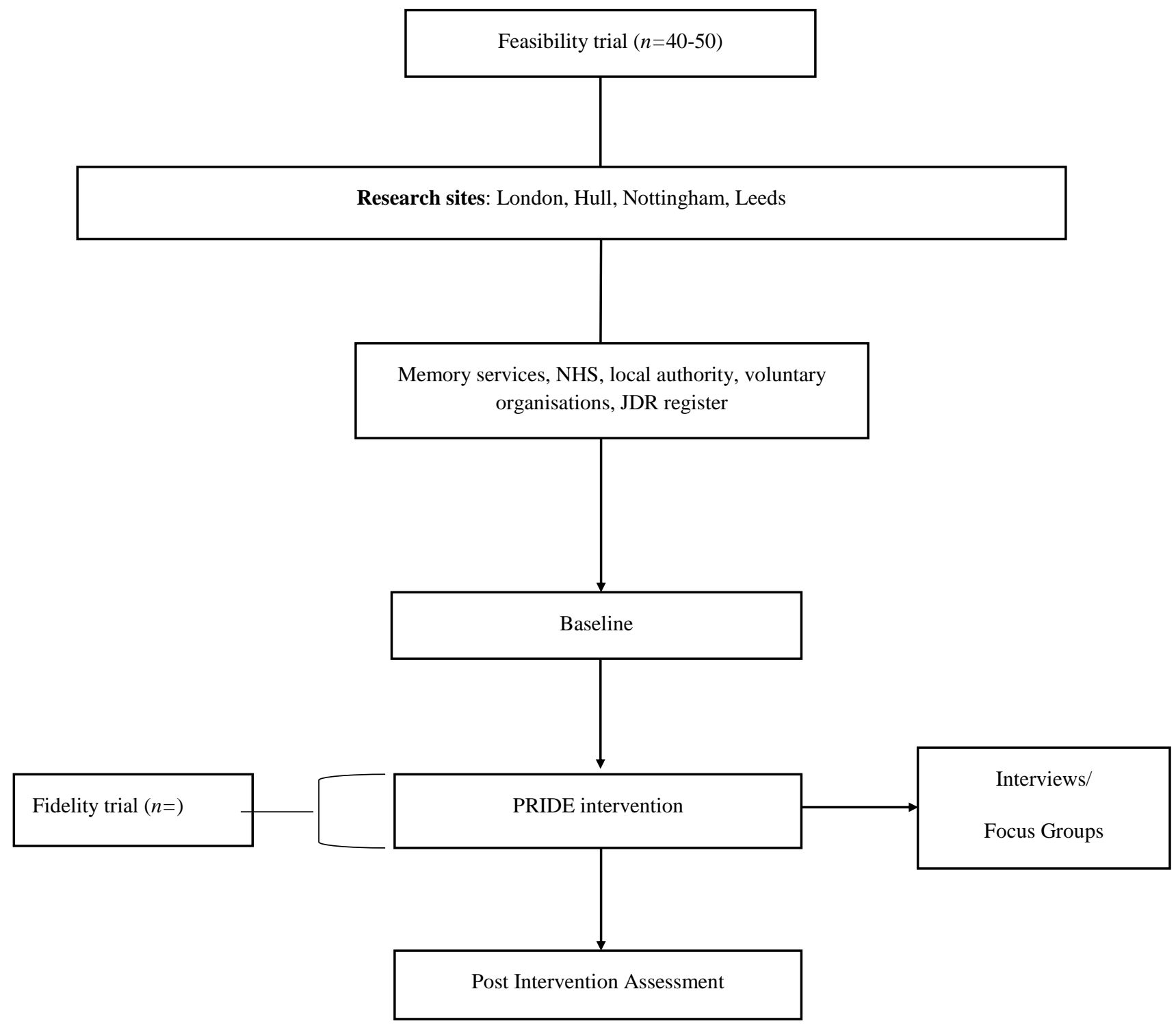

Figure 1: Participant flow through feasibility (one), fidelity (two) and qualitative (three) studies.

The intervention includes a paper based manual with resources on communication, social connections, making decisions, keeping active (mentally, physically and socially), finding a balance in activities, adjusting to receiving a diagnosis of dementia, and keeping healthy. Each section features practical information drawn from clinical guidelines, factsheets issued by organisations such as the Alzheimer's Society, and the multidisciplinary clinical and research (e.g., evidence from trials) expertise of the PRIDE work group (e.g., old age psychiatry, occupational therapy, clinical psychology). Case stories have been developed based on data from interviews and work conducted in the PRIDE social and personal constructs of dementia qualitative study. For example, how people live well with dementia as well as challenges people commonly encounter, along with suggested strategies to tackle them. The person will choose resources in the manual that are most relevant to them. DAWs will provide supplementary materials and use their knowledge of local resources and services to signpost where necessary.

The intervention consists of three sessions, each lasting about one to two hours each, delivered approximately every four weeks in the participant's home. In session one, the person will discuss important aspects of their current daily lives, how to maintain or enhance the activities/routines they value, and consider new activities they might benefit from. The person will map their social network to identify strengths and the areas where they may need extra support. Structured plans will be created and recorded to encourage the person to engage in particular activities or actions (e.g. spending 30 minutes walking twice a week). Between each session, the person and their supporter will try out strategies and activities they have planned together and record what they have 
done. In the second session, progress will be reviewed with the DAW. Plans may be refined according to the participant and supporters' experience in the first month. Barriers that prevented implementation of plans will also be identified, and solutions explored. In the third and final session, progress with be reviewed again, and a maintenance plan will be developed to encourage longterm change.

Participants will be able to receive telephone support from their DAW in between intervention sessions, which will be recorded by the DAW. DAWs will have access to support and supervision from the research team.

\section{DAW training}

All DAWs will be required to attend a day standardised training session to ensure the intervention is delivered as intended. The training package will be developed alongside the development of the intervention, based on literature, previous expert experience and consultations with people with dementia, supporters, healthcare professionals, older people and DAWs.

The training will aim to give DAWs a comprehensive understanding of the intervention. It consists of; (1) an overview and aims of the PRIDE feasibility study, (2) discussion of the role of DAWs, (3) the intervention procedure, (4) content of the PRIDE manual, and (5) helping participants identify goals, make plans and review them and problem solve. DAWs will be provided with an accompanying training manual. The training will be delivered by the same research team members across the different sites in order to increase fidelity in delivery of the intervention. The training session comprises of presentations, demonstrations of techniques and role plays. DAWs will have the opportunity to actively practise the skills and try the exercises in the manual which will consolidate learning and enable the trainers to observe their initial understanding of the concepts presented.

\section{Screening and outcome measures}

\section{Screening}

The clinical dementia rating scale (CDR) will be used to screen people with dementia. ${ }^{20}$ It rates impairment in six cognitive categories (memory, orientation, judgment and problem solving, community affairs, home and hobbies, and personal care) on a five-point scale (0-3). In order to be eligible, people with dementia will score $0-1$, indicating mild dementia. The screening will be carried out by researchers.

\section{Outcome measures for the person with dementia}

The primary outcome will be functional ability of the person measured by the Bristol activities of daily living scale (BADLS). ${ }^{29}$ The measure is completed by the supporter and consists of 20 daily-living abilities. There is evidence to suggest the BADLS shows sensitivity to change in people with Alzheimer's disease receiving anticholinesterase medication and significantly correlates with changes in the Mini-Mental State Examination (MMSE), and the Alzheimer's disease assessment scaleCog (ADAS-Cog). ${ }^{30,31}$

Secondary outcomes for the person will include; quality of life (QoL) (Dementia Quality of Life (DEMQOL), Control, autonomy, pleasure and self-realisation (CASP19); ICEpop CAPability measure for Older people (ICECAP-O), health-related QoL (EQ-5D, functional mobility (Timed up and go test), English Longitudinal Study of Aging (ELSA) self-perceived social connectedness questions, verbal fluency and learning (Hopkins verbal fluency and learning test (HVLT), cognition (Standardised mini-mental state examination (S-MMSE), self-determination and participation (Impact on participation and autonomy (IPA), and subjective independence and social engagement (Engagement and Independence in dementia questionnaire (EID-Q)). ${ }^{32-41}$ Resilience and hope will be measured using the positive psychology outcome measure (PPOM), self- management abilities will be measured by the Self-management abilities scale (SMAS-30). ${ }^{41,42}$

\section{Outcome measures for supporters}

The primary outcome for supporters will be supporter health-related QoL measured by the EQ-5D. ${ }^{36}$ The EQ$5 \mathrm{D}$ is a measure of self-reported health outcomes that is applicable to a wide range of health conditions and treatments. It consists of two parts: a descriptive system (Part I) and a visual analogue scale (VAS) (Part II). Part I of the scale consists of five single-item dimensions including: mobility, self-care, usual activities, pain/discomfort, and anxiety/depression. Part II uses a vertical graduated VAS (thermometer) to measure health status, ranging from worst imaginable health state to best imaginable health state.

QoL will be the secondary outcome for supporters and will be measured using the ICECAP-O. ${ }^{34}$

\section{Cost data}

Economic cost data on supporters' costs and participants' use of health and social care services will be gathered using the Client Services Receipt Inventory (CSRI). ${ }^{43}$ A retrospective period of three months will be examined. In particular, we will examine the completeness of the data. This will be led by the health economics team within the larger PRIDE study.

The suitability of all measures, missing data and the length of time needed to complete the assessment will be assessed. Research staff carrying out the assessments will note if there are any particular difficulties with the measures. 


\section{Fidelity of delivery and engagement}

To assess fidelity of delivery, all intervention sessions will be audio-recorded and $60 \%$ of audio-recordings will be transcribed and rated against fidelity of delivery checklists, which were developed for the study. Checklists and coding guidelines will be piloted to ensure that inter-rater agreement is achieved before using them to assess fidelity of delivery.

DAWs and participants will also be asked to complete the fidelity of delivery checklists to determine what they have delivered and what they have received. The participant fidelity checklists will also be used to assess engagement, including what participants have understood in each session and the skills they have used since the last session.

\section{Development of fidelity checklists}

To develop the checklists, previous fidelity measures were reviewed, intervention components were identified and a framework of components was developed before drafting the checklists. Feedback on the checklists was then sought from relevant public and patient involvement (PPI) members on the content and wording of the checklists and guidance. Two versions of the checklists and guidance for completing the checklists were developed for use by a) DAWs/researchers and b) participants. Different checklists were developed for each session of the intervention to capture all intervention components.

\section{Qualitative evaluation}

Interviews and focus groups will be carried out after participants and supporters have completed the intervention to determine if; (1) participants found the intervention and study procedures suitable and feasible, (2) whether they found the intervention useful and appropriate, and (3) barriers and facilitators to engagement. DAWs will also take part in interviews and focus groups to examine their experience of delivering the intervention, and barriers and facilitators to delivery.

A consensus conference will be held in order to evaluate the final draft of the manual and intervention. Participants who took part in the intervention, experts in the field including academics and healthcare professionals, as well as people with dementia and carers will be invited to attend a half-day conference. Conference attendees will be sent copies of the manual prior to the conference to give them time to familiarise themselves with it. The conference will consist of presentations on the current status of the project, followed by workshop style discussions. Attendees will be split into multidisciplinary groups to promote varied discussion from several perspectives. Notes taken during the workshop discussions will be collated and suggested amendments will be incorporated into the final manual and intervention.

\section{Evaluation of recruitment capability and retention}

Referral sources (e.g. JDR, clinician referral), along with reasons for ineligibility, refusal and retention rates will be recorded. Reasons for dropout at each stage will be examined. Examining barriers to the recruitment of participants, DAWs and research sites will help inform planning the main RCT. Recruitment rates will be calculated. The acceptability and ease of use of the CDR tool and all outcome measures will be evaluated across all sites.

We will record the organisations through which we find DAWs (e.g. Alzheimer's Society, voluntary sector or National Health Service [NHS] staff), and the type of DAWs (e.g., salary band, job description) who receive training. If DAWs attend the training but do not deliver the intervention we will record the reasons for this. The study will also evaluate the availability/capacity of DAW provider at each site.

\section{Analyses}

Descriptive analyses using means (with standard deviation) and percentages will be used to summarise the baseline characteristics of the participants. T-tests and correlations will be used to compare baseline and follow up data. The inter-rater agreement of researcher fidelity assessments will be assessed using the Kappa statistic. Fidelity of delivery and engagement will be assessed using descriptive statistics to compare across sites, providers, participants, and sessions. For example, fidelity of delivery will be calculated for each session in terms of the percentage of intervention components that were delivered as planned. A Pearson's correlation will be used to correlate the researcher, participant, and dementia advice worker ratings of fidelity. Missing data will be analysed in order to determine if there are any particular difficulties with any of the measures.

\section{DISCUSSION}

This protocol describes a mixed-methods feasibility trial of an intervention aiming to promote independence for people with mild dementia. The current study may offer a potential solution to the impoverished post-diagnostic experiences of many people with dementia and carers. ${ }^{44}$ The direction of travel in memory services in England is to offer post diagnostic support from DAWs who can signpost people to ways in which they can live well with the condition. Our study will examine whether we can inform practice in line with current evidence on how people can engage with life to keep well.

The current research about self-management programmes for people with dementia is limited. ${ }^{4,45}$ However, selfmanagement approaches based on social cognitive theory 
can help people to understand the illness and build empowerment and coping strategies. ${ }^{46}$ For people with dementia developing and utilising self-management skills may enhance resilience, facilitate reciprocity, and help the person maintain their independence, sense of identity, activities, wellbeing, and relationships. ${ }^{5}$ The PRIDE intervention addresses the call for interventions which aim to ensure the voice of the person with dementia is heard; balancing the person's needs with those of the supporter, and involving the supporter and supportive others in the process of development and implementation of self-management skills. ${ }^{47}$ The intervention is personcentred in that it provides options for the range of preferences of the person rather than using a 'one size fits all' approach. A recent study showed that menu-based programmes which can be tailored to individual needs through offering choices are preferred by people with dementia. 5

In terms of contribution to the field, this study will add to knowledge of the feasibility of structured selfmanagement interventions. By incorporating process evaluation and gathering data on fidelity, the study may provide insight into the 'active' components of this type of intervention that impact outcomes as well as identifying adaptations occurring in practice which may undermine intervention fidelity. ${ }^{48} \mathrm{We}$ anticipate this information will be useful for the subsequent planned evaluation of the effectiveness of the PRIDE intervention in a large scale RCT, particularly in minimising risk of implementation errors and thereby increasing likelihood of detection of genuine effects of the intervention if they are present. $^{49}$

Self-management interventions may address the current "care gap" supporting people living with early stage dementia indicating the development of interventions like PRIDE is worthwhile. If the PRIDE intervention is found to be effective, the package could be adopted by NHS and voluntary dementia advisory services as part of routine practice. The long term impact of effective postdiagnostic support via dementia advisory services and interventions such as PRIDE may be reductions in emergency inpatient admissions and premature care home admissions, and enhancement of service access rates in minority ethnic communities. ${ }^{50}$ The data gathered in this feasibility trial will be used to inform any necessary modifications to the PRIDE intervention and materials before the main RCT.

\section{ACKNOWLEDGEMENTS}

We would like to acknowledge the input of the larger PRIDE team, and thank the research sites and participants. http://www.institutemh.org.uk/x-research/pride.

Funding: This work was supported by the Economic and Social Research Council/ National Institute of Health [grant number ES/L001802/2]. This article presents independent research funded by the National Institute for Health Research (NIHR). The views expressed are those of the authors and not necessarily those of the NHS, the NIHR or the Department of Health. It is collaboration between University College London.

Conflict of interest: None declared

Ethical approval: The study was approved by East Midlands - Nottingham 1 Research Ethics Committee (REC) (Ref: 16/EM/0044). As a non pharmalogical intervention this is not covered by the Medicines for Human Use (Clinical Trials) Regulations 2004.

\section{REFERENCES}

1. MonizCook E, Manthorpe. Early Psychosocial Interventions in Dementia: Evidence Based Practice, London, Jessica Kingsley 2009.

2. European Union (EU) Joint Programme Neurodegenerative Diseases (JPND): JPND Research Strategy. 2012. Available at: http://www.neurodegenerationresearch.eu. Accessed on 4 November 2017.

3. Katsuno T. Dementia from the inside: how people with early-stage dementia evaluate their quality of life. Ageing Soc. 2005;25:197-214.

4. Martin F, Turner A, Wallace L, Choudhry K, Bradbury N. Perceived barriers to self- management for people with dementia in the early stages. Dementia. 2012;12(4):481-93.

5. Mountain GA, Craig CL. What should be in a selfmanagement programme for people with early dementia? Aging Ment Health. 2012;16(5):576-83.

6. Muo R, Schindler A, Vernero I, Schindler O, Ferrario E, Frisoni G. Alzheimer's diseaseassociated disability: An ICF approach. Disab Rehab. 2005;271405-13.

7. Kuiper JS, Zuidersma M, Voshaar RCO, Zuidema SU, van den Heuvel ER, Stolk RP, et al. Social relationships and risk of dementia: A systematic review and meta-analysis of longitudinal cohort studies. Ageing Res Rev 2015;22:39-57.

8. Yates LA, Ziser S, Spector A, Orrell M. Cognitive leisure activities and future risk of cognitive impairment and dementia: systematic review and meta-analysis. Int Psychogeriatr. 2016;28(11):1791806.

9. Hogervorst E, Clifford A, Stock J, Xin X, Bandelow S. Exercise to prevent cognitive decline and Alzheimer's disease: for whom, when, what, and (most importantly) how much. J Alzheimers Dis Parkinsonism. 2012;2-3.

10. Low L-F, Swaffer K, McGrath M, Bradley H. Do people with early stage dementia experience Prescribed Disengagement? A systematic review of qualitative studies. Int Psychogeriatr. 2017.

11. Reamy AM, Kim K, Zarit SH, Whitlatch CJ. Understanding discrepancy in perceptions of values: individuals with mild to moderate dementia and their family caregivers. Gerontologist. 2011;51(4):475-83. 
12. Smebye KL, Kirkevold M, Engedal K. How do persons with dementia participate in decision making related to health and daily care? A multicase study. BMC Health Serv Res. 2012;12(1):241.

13. Tyrrell J, Genin N, Myslinski M. Freedom of choice and decision-making in health and social care. Views of older patients with early-stage dementia and their carers. Dementia. 2006;5(4):479-502.

14. Boyle G. She's usually quicker than the calculator': financial management and decision-making in couples living with dementia. Health Soc Care Com. 2013;(5):554-62.

15. Brody EM, Kleban MH, Lawton MP, Silverman HA. Excess disabilities of Mentally impaired aged: Impact of individualized treatment. Gerontologist. 1971;11(2 Part 1):124-133.

16. Spector A, Orrell M. Using a biopsychosocial model of dementia as a tool to guide clinical practice. Int Psychogeriatr. 2010;22(6):957-65.

17. Mountain GA. Self-management for people with early dementia: An exploration of concepts and supporting evidence. Dementia: London. 2006;5:429-46.

18. Knapp M, Black N, Dixon J, Damant J, Rehill A, Tan S. Independent Assessment of Improvements in Dementia Care and Support since 2009. London: Policy Innovation Research Unit and NIHR School for Social Care Research, LSE and LSHTM. 2014.

19. American Psychiatric Association. Diagnostic and statistical manual of mental health disorders, 4th ed. Washington DC: APA. 1994.

20. Morris JC. The Clinical Dementia Rating (CDR): current version and scoring rules. Neurology. 1993;43(11):2412-4.

21. Department of Health. Mental Capacity Act, London, HMSO. 2005.

22. Craig P, Dieppe P, Macintyre S, Michie S, Nazareth I, Petticrew M. Developing and evaluating complex interventions: the new Med Res Council guidance. BMJ. 2008: 337.

23. McDermott O, CharlesworthG, Hogervorst E, Stoner C, Moniz-Cook E, Spector A, et al. Psychosocial interventions for people with dementia: a synthesis of systematic reviews. Aging and Mental Health, 2018: 1-11.

24. Birt L, Poland F, Csipke E, Charlesworth G. Shifting dementia discourses from deficit to active citizenship. Chapter in Higgs $\mathrm{P}$ and Gilleard C editors: Ageing, dementia and the social mind. Wilery Blackwell, Oxford; 2017.

25. Rafnsson S, Orrell M, d'Orsi E, Hogervorst E, Steptoe A. Loneliness, Social-Relationships and the Risk of Dementia: Prospective Findings from the English Longitudinal Study of Ageing. J Gerontol B Psychol Sci Soc Sci. 2017.

26. Hogervorst E. Healthy lifestyles to prevent dementia and reduce dementia symptoms. Working with Older People. 2017;21:31-9.

27. Xavier AJ, d'Orsi E, Ramos LR, Sigulem D, dos Santos JB, Quialheiro A, Olivieri G et al. Cognitive stimulation and rehabilitation mediated by computers and internet: a controlled study. Alzheimers Dem. 2010;6(4):S163.

28. Baltes PB. On the incomplete architecture of human ontogeny: Selection, optimization, and compensation as foundation of developmental theory. Am Psychol. 1997;52(4):366.

29. Bucks RS, Ashworth DL, Wilcock GK, Siegfried K. Assessment of activities of daily living in dementia: development of the Bristol Activities of Daily Living Scale. Age Ageing. 1996;5(2):113-20.

30. Folstein MF, Folstein SE, McHugh PR. ("Minimental state". A practical method for grading the cognitive state of patients for the clinician. J Psychiatr Res, 1975;12(3):189-98.

31. 31. Byrne LM, Wilson P, Bucks RS, Hughes AO, Wilcock GK. The sensitivity to change over time of the Bristol Activities of Daily Living Scale in Alzheimer's disease. Int Journal Geriatric Psy. 2000;5(7):656-61.

32. Smith SC, Lamping DL, Banerjee S, Harwood A, Knapp M, et al. Measurement of health-related quality of life for people with dementia: Development of a new instrument (DEMQOL) and an evaluation of current methodology. Health Technol Assess. 2005;9(10):1-112.

33. Hyde M, Wiggins RD, Higgs P, Blane DB. A measure of quality of life in early old age: the theory, development, and properties of a needs satisfaction model (CASP-19). Aging Ment Health. 2003;7:186-94.

34. Coast J, Flynn TN, Natarajan L, Sproston K, Lewis J, Louviere JJ, Peters TJ. Valuing the ICECAP capability index for older people. Soc Sci Med. 2008;67(5):874-82.

35. EuroQoL Group. EuroQoL: A new facility for the measurement of health related quality of life. Health Policy. 1990;16:199-208.

36. Podsiadlo D, Richardson S. The timed" Up \& Go": a test of basic functional mobility for frail elderly persons. J Am Geratric Soc. 1991;39(2):142.

37. Hughes ME, Waite LJ, Hawkley LC, Cacioppo JT. A short scale for measuring loneliness in large surveys results from two population-based studies. Res Aging. 2004;26(6):655-72.

38. Hogervorst E, Combrinck M, Lapuerta P, Rue J, Swales K, Budge M. The Hopkins verbal learning test and screening for dementia. Dementia Geriatr Cogn. 2002;13(1):13-20.

39. Molloy DW, Alemayehu E, Roberts R. Reliability of a standardized mini-mental state examination compared with the traditional mini-mental state examination. Am J Psychiatry. 1991;148(1):102-5.

40. Hammar IO, Ekelund C, Wilhelmson K, Eklund K. Impact on participation and autonomy: test of validity and reliability for older persons. Health Psychol Res. 2014;2(3):1825.

41. Stoner CR, Long M, Csipke E, Spector A. The development and preliminary psychometric properties of two positive psychology outcome 
measures for people with dementia: the PPOM and the EID-Q. BMC Geriatr. 2017;17:72.

42. Schuurmans H, Steverink N, Frieswijk N, Bunk BP, Slaets JP, Lindenberg S. How to measure selfmanagement abilities in older people by self-report. The development of the SMAS-30. Qual Life Re. 2005;14(10):2215-28.

43. Beecham J, Knapp M. Costing psychiatric interventions. In Measuring Mental Health Needs. G. Thornicroft editor. London: Gaskell; 1992: 163183.

44. Manthorpe J, Samsi K, Campbell S, Abley C, Keady J, Bond J, Watts S, Robinson L, Warner J, Iliffe S. From forgetfulness to dementia: clinical and commissioning implications of diagnostic experiences. Brit J Gen Prac. 2013;63(606) e69-e75.

45. Toms GR, Quinn C, Anderson DE, Clare, L. Help Yourself: Perspectives on Self-Management From People With Dementia and Their Caregivers. Qual Health Res. 2014;25(1):87-98.

46. Bandura A. Self efficacy: the exercise of control. New York: Freeman. 1997.

47. Quinn C, Clare L, Woods B. The impact of the quality of relationship on the experiences and wellbeing of caregivers of people with dementia: a systematic review. Aging Ment Health. 2009;13(2):143-54.

48. Moore GF, Audrey S, Barker M, Bond L, Bonell C, Hardeman Q, Moore L, et al. Process evaluation of complex interventions: Medical Research Council guidance. Brit Med J. 2015; 50: h1258.

49. Vernooij-Dassen M, Moniz-Cook E. Raising the standard of applied dementia care research: addressing the implementation error. Aging Ment health. 2014;8(7):809-14.

50. Knapp M, Black N, Dixon J, Damant J, Rehill A, Tan S. Independent assessment of improvements in dementia care and support since 2009. Policy Innovation Research Unit (PIRU), 2014. Available at: http://researchonline.lshtm.ac.uk/2391582/1/ 2014-9\%20Assessing\%20Dementia\%20Report.pdf Accessed on 15 Nov 2017.

Cite this article as: Csipke E, Yates L, Cook EM, Leung P, Charlesworth G, Walton H, et al. Promoting independence in dementia: protocol for a feasibility trial of the PRIDE intervention for living well with dementia. Int J Clin Trials 2018;5(4):177-85. 